\title{
Initial Experience with the Volta Phase Plate
}

\author{
Michael Marko ${ }^{1}$, and Chyongere Hsieh ${ }^{1}$ \\ 1. Wadsworth Center, New York State Department of Health, Empire State Plaza, Albany, NY 12201
}

Specimens prepared solely by vitreous freezing facilitate high-resolution imaging of macromolecules in-situ and in a near-native state. However, such samples are weakly scattering, suffering from low contrast and requiring phase-contrast imaging. In addition, high-resolution information is limited by electron-radiation damage. Cryo-TEM technology has steadily been improving so that improved image contrast can be obtained without increased electron dose.

After cryo-TEM specimen stages became common, the next advance was implementation of zero-loss energy filtering, approximately doubling contrast without increasing the electron dose [1]. Starting in 2000 with the renaissance of phase-plate imaging, the oscillating contrast-transfer function of traditional defocus-phasecontrast imaging could be avoided, providing near-uniform transfer of information over a wide range of spatial frequencies [2]. Very recently, practical implementation of direct-electron detectors have led to great advances $[3,4]$, due to their improved efficiency over previous cameras or photographic film, and also due to a rapid frame rate, such that alignment of a stack of frames can correct for specimen movement during exposure [5,6,7].

We are concerned with optimizing phase-contrast imaging. While there are many means to do so, as reviewed by Glaeser [8], we are currently concentrating on phase plates. The Zernike phase plate (ZPP) proved itself in early biological applications $[9,10]$ and has become the standard for practical application [11]. However, the introduction of the Volta phase plate (VPP) [12] promises to replace the Zernike for routine applications [e.g. 13]. A Volta phase plate has the same configuration as a "hole-free phase plate" (HFPP) [14], and can be easily constructed in any TEM lab by simply placing a thin $(5-15 \mathrm{~nm})$ carbon film over an objective aperture disc. Unlike the ZPP, there is no requirement to create, and then accurately center, a small central hole. A VPP must be operated above $200^{\circ} \mathrm{C}$, while the HFPP operates at room temperature. Images made using a VPP or HFPP do not contain the fringe artifacts, which are a result of the abrupt edge of the central hole in the ZPP.

We have been using ZPPs in our JEOL JEM-3200FSC/PP cryo-TEM for some time. This TEM is equipped with JEOL's airlock-type heated phase-plate holders [15] in both objective aperture and selected-area (SA) diffraction-plane positions, and it has a special transfer lens to effect a three-times enlargement of the objective-lens back-focal plane at the SA position. For cryo-TEM low-dose imaging, our TEM has a coarse stage piezo that can move the specimen along the tilt axis to reach the low-dose focus/tracking position.

We realized that it would be simple to use a VPP or HFPP in our TEM, and indeed the results so far with the VPP are excellent (Fig. 1). We collect tomographic tilt series with a modified version of SerialEM [16] that employs our special stage piezo. However, a simple firmware modification by JEOL allows access to the focus/tracking position by means of image shift and beam shift, while maintaining parallel illumination (i.e. the phase-plate "on plane" condition), but without any beam tilt that would cause displacement of the beam from the active center-point on the phase plate. We use JEOL's standard phase-plate positioning system [15] to select and recall the best VPPs on our multi-hole objective aperture. Tilt-series collection proceeds automatically, without the need to re-center the VPP active area.

Our TEM is ideally configured to test a variety of phase-shifting devices. We will compare thin-film, holefree phase plates at both room temperature and above $200^{\circ} \mathrm{C}$ (an HFPP / VPP comparison). We have also started to test ZPPs in the "transfer mode" (SA position) to investigate the effects of a lower cut-on frequency 
afforded by the longer effective objective-lens focal length (Fig. 2). While low-frequency contrast is clearly greater than from a VPP at the normal objective aperture position, the typical artifacts due to the abrupt cut-on of the ZPP are apparent, although they could be reduced by filtering [17,18]. We have also found that a VPP functions in "transfer mode", and we are investigating whether or not this results in improved contrast at very low spatial frequency. [19]

References

[1] R Grimm et al, J. Microsc. 190 (1998), p. 339.

[2] R Danev et al, Ultramicroscopy 109 (2009), p. 312.

[3] N Grigorieff eLife 2 (2013), p. e00563.

[4] W Kühlbrandt, eLife 3 (2014), p. e03678.

[5] B Bammes et al, J. Struct. Biol. 177 (2012), p. 589

[6] X Li et al., Nat. Methods 10 (2013), p. 584.

[7] G. McMullan et al, Ultramicroscopy 109 (2009), p. 1126

[8] RM Glaeser, Rev. Sci. Instrum. 84 (2013), p. 111101.

[9] R Rochat et al, J. Virol. 85 (2011), p. 1871.

[10] K Murata et al, Structure 18 (2010), p. 903.

[11] M Marko et al, J. Struct. Biol. 184 (2013), p. 237.

[12] R Danev et al, PNAS USA 111 (2014), p. 15635.

[13] S Asano et al, Science 347 (2015), p. 439.

[14] M. Malac et al, Ultramicroscopy 118 (2012), p. 77.

[15] W Dai et al, Nat. Protocols 9 (2014), p. 2630.

[16] http://bio3d.colorado.edu/SerialEM/

[17] H Sui et al, Microsc. Microanal 20 (2014), p. 234.

[18] R Danev and K. Nagayama Ultramicroscopy 111 (2011) p. 1305.

[19] Thanks to S. Motoki of JEOL USA for TEM support, D. Mastronarde of UC Boulder for SerialEM support, and F. Maley of Wadsworth Center for T4 phage. Supported by NIH grant GM103555 (to M. Marko).

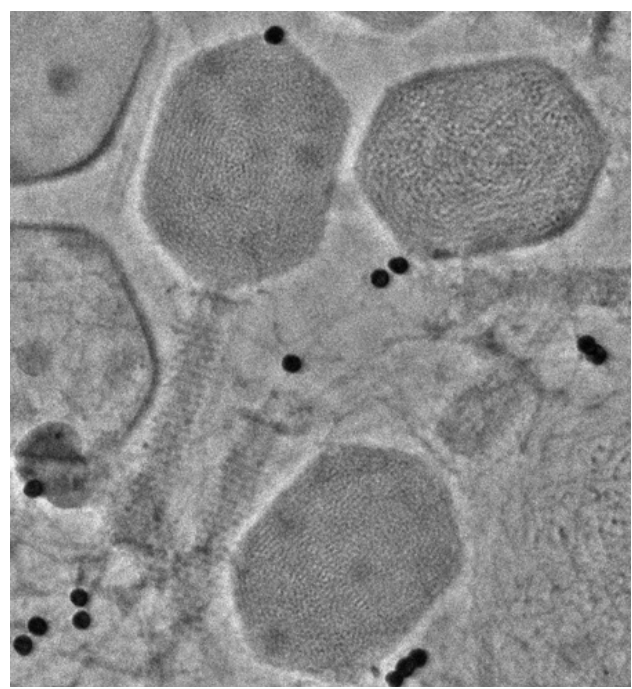

Figure 1. T4 phage with $10-\mathrm{nm} \mathrm{Au}$ particles. VPP Cryo-TEM at $300 \mathrm{keV}$ with in-column energy filter and $\mathrm{K} 2$ Summit camera in counting mode.

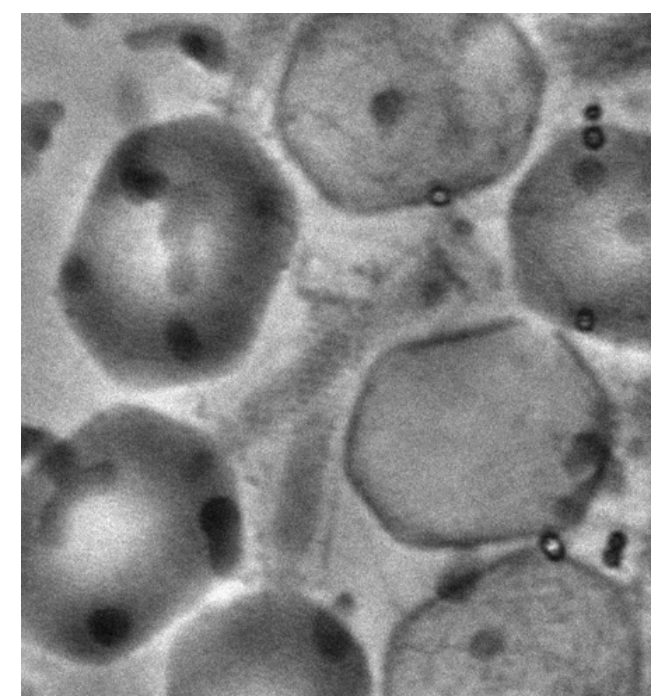

Figure 2. T4 phage imaged as in Fig 1, but with ZPP at $3 \mathrm{X}$ greater focal length. Hole size $700 \mathrm{~nm}$. Note increased contrast of tails, and "fringe" artifacts on the left. 